\title{
Agent-based On-line Analytical Mining in CRM System
}

\author{
Chen Feng, Wang Cong, Lin Jie \\ Research Center of Intelligence Sci.\&Tech. Engineering Dept. of \\ Beijing University of Posts and Telecommunications, Beijing 100876, China \\ Email: lotus1024@tom.com,wangc@bt-t.com, benlj@163.com
}

\begin{abstract}
The OLAM (On-Line Analytical Mining) model based on agent is proposed in this paper, and a generalized data warehouse built on Multi-Bases Cooperation Technique is proposed, which makes DM applicable not only on structured data, but also on complicated data such as text, knowledge and so on. Furthermore, I apply the theory of Union-IADS to construct a data space that comprises the generalized data warehouse mentioned above and data marts. In the end, to build an intelligent CRM systent, I integrate the agent-based OLAM model and the data space into a unifying framework, which is a new try on building a CRM system.
\end{abstract}

Index Terms-AI (Artificial Intelligence), CRM (customer relationship management) , IADS (Intelligent Autonomous Decentralized Systems), OLAM (on-line analytical mining), DM (data mining)

\section{INTRODUCTION}

$\mathrm{C}$ urrent CRM systems mostly utilize OLAP analyzing tools or data mining (DM) tools, but the magnanimity data faced in CRM systems and the high data processing efficiency requested by end-users can not be managed perfectly. In order to deal with the problem proposed above, this paper improves current CRM on two aspects: firstly, implement the seamless combination of OLAP and DM; secondly, apply the theory of Intelligent Management System (IMS).

Although OLAP analyzing can provide users the view of different angles and different abstract ranks, because the knowledge of user's needs is not deepened and in an all-round way in advance, the view lacks the expected dimensions, and the results from different views may not be the same, prone to misleading. The users need to make a large number of "data salvage" work and guess the correct result just according to concrete data, and may still omit the important model and relations among the data [4].

There are also dense integral relations among each data mining method [1]. The data mining function is used in the model type, which is looked for in appointed data mining tasks. The main data mining tasks are as follows: concept description, association, classification, prediction, clustering, time-series

0-7803-8125-4/03/\$17.00 C 2005 IEEE analysis, etc. Actually, classification analysis is the factor analysis under a certain prerequisite. Association rules offer horizontal and vertical relations. Clustering analysis can offer the foundation of abstract conceptions for the broadly related rules. And all these can be represented or analyzed by OLAP, and the result of the data mining can guide the OLAP multi-dimensional model.

Meanwhile, data warehouse can be utilized in OLAP as well as the DM; OLAP is as one kind of the data analytical tools, which is not isolated and must cooperate with the other data mining tools; The effective data mining needs OLAP to carry on the surveying type data analysis; By integrating OLAP with various data mining tools, on-line analytical mining offers flexibility for the data mining function expected by the users and automatic adaptation of the data mining tasks [3].

From the analysis above, this paper proposes an agent-based OLAM model firstly, and then applies the model into CRM system, which embodies the advantages of OLAM model that is based on intelligent agent.

\section{RESEARCH ON AGENT-BASED OLAM}

The concept of OLAM is based on multi-dimensional and multi-level data view [4]. However, OLAM is not a simple combination of OLAP and DM, most OLAM models researched now are all adopting the data cube to be the storage structure of data and computing basic in data mining, without considering the whole searching space of our problems, so that we propose an agent-based model that can solve this problem.

\section{A. Agent-based OLAM mode}

As for the conception above we design an OLAM model based on agent, which could be called OLAM engine shown as fig. 1).

Comparing the two models in fig. 1 and fig. 2 , there are the following differences: the former adopts advantages from both OLAM and DM sufficiently, which makes data mining fulfilled in comprehensive multi-dimensional and muiti-level space; the former is designed based on Multi-Bases Cooperation Technique, while the latter is based on simple data warehouse. Constructing data cube based on Multi-Bases Cooperation Technique is not a simply disposal of data, abstracting associated data and analysis of polysemy on the raw data, but 
may often involve the analysis of more dimensions with finer granularities, or involve the discovery-driven exploration of multi-feature aggregations on the data cube; For the data utilized in the OLAM engine is the one mine data in multi-dimension and multi-level, the decision-making agent in the former model shall have more powerful decision-making capabilities. What we should emphasize is the former is constructing on Multi-Bases Cooperation Technique, so it introduces information science in the processing of data mining.
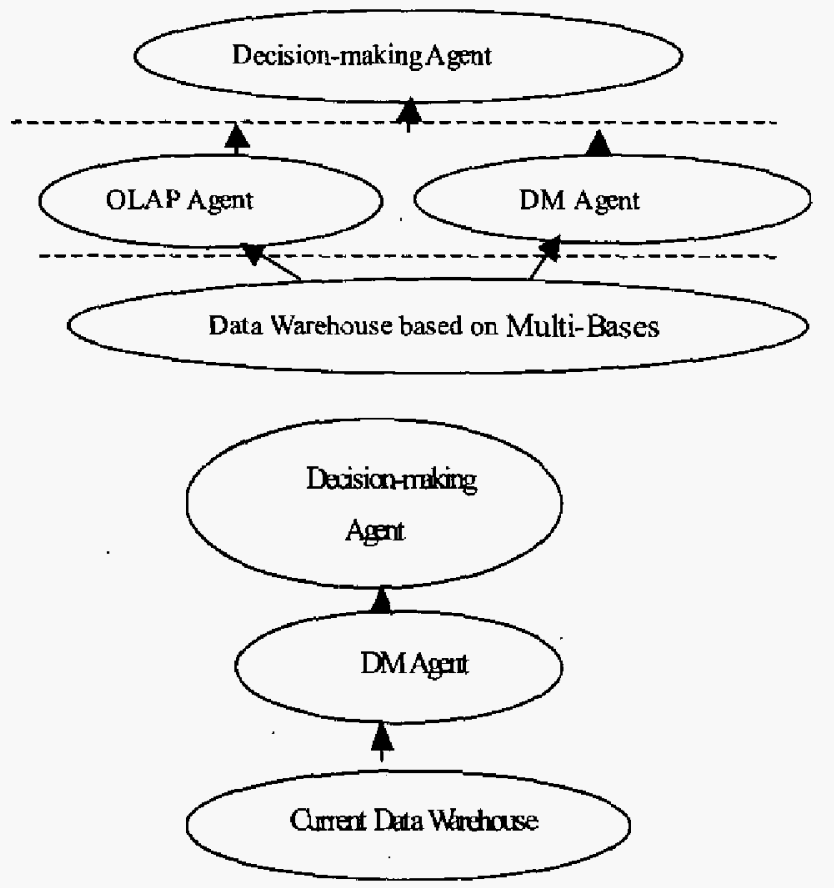

Fig. 2. DM model based on agent [5]

\section{B. Research on Agent-based OLAM Model}

I) Build a generalized data warehouse based on Multi-Bases - Concept

Constructing a data warehouse based on Multi-Bases Cooperation Technique [8], which is called generalized data warehouse in this paper. Generalized data warehouse includes traditional data such as number and character, and also includes complicated data such as text. As a matter of fact, the useful information contained in the complicated data is 3 to 5 times as much as the traditional data. With the popular of e-commerce, the quantity of complicated data is huge in web sites. So the ability to abstract information from conplicated data is to be the business demand. Because generalized data warehouse contains complicate data, we quote $\mathrm{AI}$ to do semantic and syntax analyze in it, however, how to use AI to analyze complicate data in the data cube is a great difficult. In order to deal with the problem, we introduce algebra for on-line analytical processing data cube, which can be the theoretical foundation of semantic specification of data warehousing and OLAP manipulations. The description of the algebra is as follow ${ }^{[2]}$ :
(1) The algorithm describing of data cube cube $=($ dom,$d$, mdom $, m, f, a g g r)$

(2) $d o m=d o m_{1} \times \ldots \times d o m_{n}, N>0$, which is called the domain of dimension, $\operatorname{dom}_{i}(1 \leq i \leq n)$ is the domain of dimension

(3) $D=\left\{d_{1}, \ldots d_{n}\right\}$, Which is called the set of domain, $d_{i}(1 \leq i \leq n), \quad d_{i}$ is the identifier of $\operatorname{dom}_{r}(1 \leq i \leq n)$

(4) $M d o m=m d o m_{1} \times m d o m_{m}, m>0$, which is called the domain of guidelines, $\operatorname{mdom}_{i}(1 \leq i \leq n)$ is the domain of guidelines

(5) $M=\left\{m_{1} \ldots m_{m}\right\}$, Which is called the set of guide lines, $m_{i}$

$(1 \leq i \leq n)$ is the identifier of $\operatorname{malom}_{\mathrm{i}}(1 \leq i \leq n)$

(6) $f: D o m \rightarrow M d o m$ Is the mapping from Dom to Mdom which is called the radix of data cube;

(7) aggr Is gather function in Mdom

While the value land of dimension and identifier is determined, data cube can be expressed as $c u b e=(d o m, d$, mdom $, m, f, a g g r)$

With the algebra of database introduced above, we can analyze text data in the generalized data cube based on the algebra, for example, the conversion of guide line and dimension, selection sets that have the same characteristics and so on.

- Functions

The generalized data warehouse not only helps to build a powerfil warehouse for the on-line analytical mining, but also helps to make preparations for constructing data marts.

- Characteristics

Generalized data warehouse contains complicated data such as text, so it is a dynamic information storehouse. In the storehouse, all kinds of information from commerce can be receipted and understood. The "Multi-Bases Cooperation" (MBC) Technique was developed for implementation of Intelligent Management Systems (IMS), One of them is shown in Fig.3 [8].

In Fig 3,

MBC-Multi-Bases Coordinator

DB-Data Base

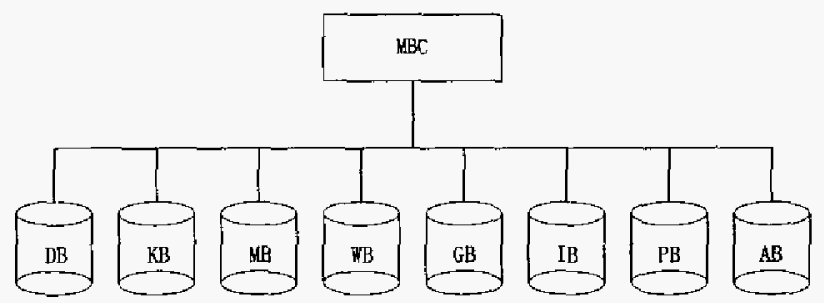

Fig. 3. Multi-Bases Cooperation System

$\mathrm{KB}-$ Knowledge Base

$\mathrm{MB}$-Model Base 
WB-- Way Base

GB-Graph Base

IB-Image Base

PB-Phoneme Base

$\mathrm{AB}$-Animation Base

The MBC technique can be applied to implement the Multi-Media Information Base for IADS.

2) OLAM Agent

- Concept

The OLAM agent integrates OLAP and data mining into a unified framework, and carrying on data mining in generalized data warehouse.

- Functions

OLAM agent leads to a data mining system, which may tour around the selected data space at will and mine knowledge with multiple, integrated mining tools. It mines in the generalized data warehouse space according to actual need and indications from customers, and fulfilling the identification of data model to discover new models or rules. OLAM agent uses various mining methods to analyze data in database, especially the information technology which provides data mining can do anywhere in the cube, such as the life style of customer, taste of customer, customer's purchase frequency, the customer belongs to which group; the common characteristics of different customers; the visiting circumstantialities of the page; the circumstantialities of clicking advertisement etc.

- Characteristics

Multi-dimensional association rule means the association rule exists in dimensions of properties, and multi-level association rule means the rules mined from different notional layers [4]. As items in each set come from different dimensions, the arisen frequency of the set can get easily, the division of notional layers in dimensions has been comprised in structure based on generalized data warehouse, so on-line analytical mining in multi-dimension and in multi-level based on generalized data warehouse will improve mining efficiency greatly.

3) Decision-making Agent

- Concept

Decision-making agent makes use of information that acquired from the OLAM agent in evaluating and verifying, and then applies confirmed conclusion and models in practice.

- Functions

Firstly, decision-making agent computes customer profit ability and forecast customer intending profit ability according to mining result. By making the model of customer profits ability, building market strategy that is based on the model. Secondly, making a model that can keeps customers from transferring to the other products. We are usually use decision tree in making model. Thirdly, it will make decision support for cross marketing. Fourthly, it can help to build a model for attracting customer by data mining and statistical method. Lastly, it can make use of information mining technology and knowledge discovery technology to supply intelligent decision support.

\section{DESIGN AND APPLICATION OF THE CRM SYSTEM BASED ON} IADS

The OLAM Engine has the function of on-line analytical mining. This paper applies this OLAM engine in CRM system that is based on IADS theory, and analyzes the characteristics of the new system. The analysis below contains two-parts: the theory of how to apply IADS in CRM system; the application of OLAM engine based on Agent in CRM system.

The IADS can be considered as the development based on the combination of DAI (Distributed Artificial Intelligence) and ADS (Autonomous Decentralized System), which can be represented as: DAI + ADS $\rightarrow$ IADS. By means of the Agent-Oriented methodology, there had developed three kinds of IADS architecture are suggested as follows, which are Union- IADS, Market- IADS and Group-IADS. The Data Warehouse in the new CRM system is based on Union- IADS, and the advantages of applying Union- IADS in the system will be discussed. The framework of Union- IADS is shown as fig. 4 .

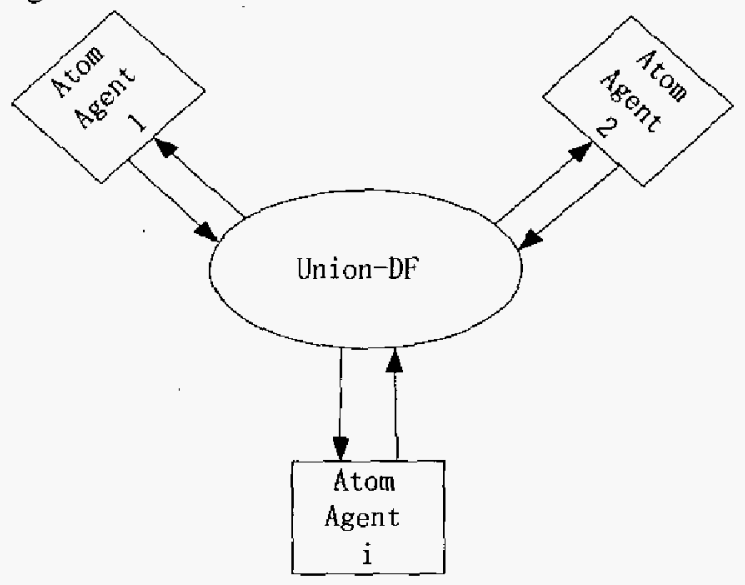

Fig. 4. The Union IADS consist of:

\section{- Union DF}

The Union Data Field is the Union Media Network (UMN) supported by the Union Information Base (UIB), Such as the "Extranet" among the various agents of the Union.

\section{- Atom Agents}

The Atom Agent $\mathrm{i}(\mathrm{i}=1,2 \ldots \mathrm{n})$ may be an Autonomous Control Agent (ACA) or an Autonomous Cootdination Multi-Agent System (ACMAS).

Data mining in most of CRM system is based on separated data warehouse or based on separated data marts, although some of them are based on multiple data marts.

However, the CRM system discussed in the paper is based on Union-IADS, which is built on a special data space comprises by data warehouse and multiple data marts. The data space is a dynamic data union, which can change its mining space according to different mining purpose. Data warehouse as a union information base, not only storage data from the data marts around it, but also storage data from outside world. And every data matt in the system is an atom agent, which can come in and go out the Union DF freely. 


\section{A. System structure analysis}

This CRM system is shown as fig.5. It has feedback function comprises five parts: data space based on Union-IADS, on-line analytical Mining engine, results informing and decision feedback.

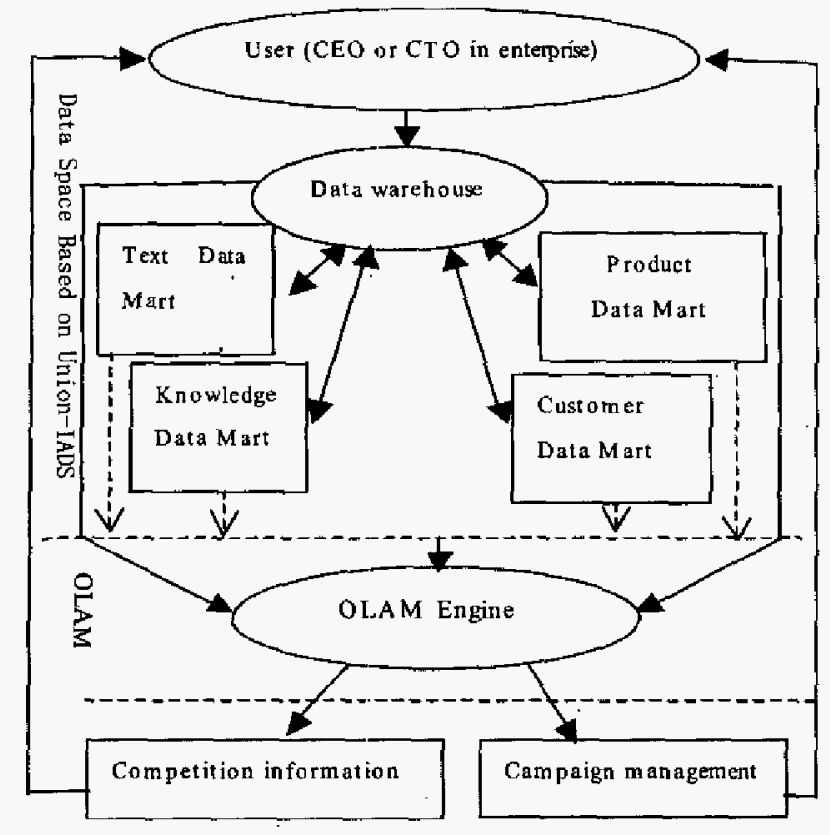

Fig.5. CRM system built by OLAM engine

\section{1) OLAM engine}

The data resource for on-line analytical mining comes from data warehouse and data marts. We should choose the right data resource according to the different needs. If user wants to mining information about specific subject, he should choose data mart as data resource, or else, he would better choose data warehouse to be data resource. After choosing the right data resource, OLAM engine begins to process structured data and complicated data such as text, however the processing of transformed text data into a unified structure and format is difficult. OLAM engine performs analytical mining in data cubes in a similar manner as an OLAP engine performs on-line analytical processing. OLAM Agent adopts data mining methods in multi-dimension and multi-level data space to analyze data on database. In order to add or replace mining methods conveniently, we can make methods to be transferring base and organize all mining methods with plug-ins. This will make OLAM extended and selective. We may also utilize selected items to select mining methods or let mining agent select appropriate algorithms directly according to different demand.

2) Results informing

Evaluates and verifies observed regulations and patterns through OLAM agent, and applies confirmed conclusions in practice. It supplies users ordered information, verification of personal page, advertisement design, warning of valuable information, intelligent competition information in business, marketing strategy etc.

\section{3) Decision feedback}

A data mining process may disclose that the dimensions and/or measures of a constructed cube may not be appropriate for data analysis and thus suggest a refined design of the data cube, which may in turn improve the quality of data warehouse and data mart construction.

4) The performances of the data space based on Union- IADS are:

- The Union- IADS is a Multi-Agent System (MAS) with Distributed Artificial Intelligence (DAI), and Autonomous Controllability and Coordinability.

- Every Atom Agent of the Union can come in and go out the Union DF freely, so that the requirements about On-line Expansion, Fault Tolerance and On-line Maintenance can be satisfied.

- The cooperation and coordination of union is achieved by consultation and communication among the various Atom Agents by Union DF.

- The Autonomy of each Atom Agent is constrained relatively by the agreement or protocol of the union.

- Each Atom Agent can satisfy: Equality, Locality and Self-containment and realize: Autonomous control and Autonomous coordination.

- The design and implententation of Union IADS can obtain some heuristic knowledge from the human union.

The emphasis of a data mart is on meeting the specific demands of a particular group of knowledge users in terms of analysis, content, presentation, and ease-of-use. Users of a data mart can expect to have data presented in terms that are familiar. While it is necessary to know data mart is a decentralization of data. Because data marts have so many merits such as subject is specific, mining data is less, mining speed is fast and so on, which becomes an important data resource for data mining ${ }^{[3]}$.

\section{B. Characteristic analysis}

The CRM system mentioned above has 4 characteristics: firstly; constructing data space based on the theory of UnionIADS, which make the mining agent can choose data source. Secondly, introducing information technology to analyze complicated data such as text, and introducing business intelligence in the course of data mining. Thirdly, introducing the agent characteristics such as autonomy, activity, sensibility, reactivity, and mobility into CRM syslems. Fourthly, introducing feedback mechanism into CRM system (see Fig.6), so user's feedback information can be sent to the system in time that will in turn improve the quality of data warehouse and data marts. Applying OLAM technique and feedback mechanism into CRM system is a trying a promising trend.

\section{CONCLUSIONS AND PRospects}

The applications of OLAM in CRM system based on agent were described above. OLAM really strengthens the analysis function in data cube. In particular, applying OLAM engine in CRM system will surely provide useful rules in the overwhelming amount of customer information, competitive information and the quick upgrading of information. It can provide innovative operation model for the industries and 


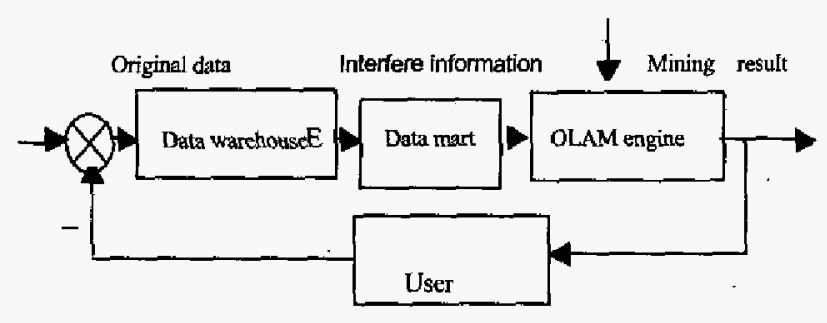

Fig. 6. Feedback principle

provide balanced operation platform for the branches of the industries. However, the OLAM technology is still in its early stage of development and there are yet many aspects, which await further study and research [7].

In particular, this paper introduces agent technology into OALM, provides an agent-based OALM model. It utilizes its capacities of knowledge (field knowledge, communication knowledge, control knowledge), goal and reasoning, decision-making, plan, ability of controlling etc., and the attributes of automity, sociality, reactivity, mobility, activity etc., thus the intellectuality of the whole mining course is realized. Meanwhile, this paper proposes the concept of generalized data warehouse, which will make a great difficult in building data cube.

In the information era, we must fully utilize the information resources of the enterprises, and shift from the product-oriented management model to the customer-oriented management model. By using the data mining technology to analyze the customer's characteristics, exploring the running regularity of enterprises and their corresponding markets and constantly improve the economic benefits will be the only way of enterprise's development.

\section{REFERENCES}

[1] Lei. Shi, Discuss of Exploring Data Ming Model, Henan China Science, 2000.3

[2] Jian Pei, Wei Cai, Chang Zhao, Shiwei Tang, Dongqing Yang, An Algebra for On line Analytical Processing Data Cube, JOU RNAL, O F SO FTWARE, Volt 10, No.6 1999

[3] Jiguang.Cao, The Research on On-line Analytical Mining Technology, Zhejiang University

[4] Qiong. He, Tanren Liu, Ping Guo, Data Ming Algotithm of Association Rules in Multi-dimension and Multi-level Data Cube, computer applications, 2004.3

[5] Alex Berson, Stephen Smith, Kurt Thearling, Building Data Mining Applications for CRM

[6] J.W. Han, OLAP Mining: An Integration of OLAP with Data Mining [’], Proc. 1997 IFIP Conference on Data Semantics (DS - 7), Leysin, Switzerland, 1997.

[7] M. S. Chen, J.W. Han, and P. S. Yu, Data Mining: An Overview from a Database Perspective []].

[8] Tu Xuyan, Tang Tao. Intelligent Autonomous Decentralized System (IADS). Proceedings of the 2nd International Workshop of IEEE Computer Society on Autonomous Decentralized System, 2002: [0-15
Chen Feng, a postgraduate in CITSR (Center for Intelligence Technology and Science Research) in Bejijing University of Posts and Telecommunications, major in Cybernetics and lntelligence science, and at present, focus on Network technology and Information Systems.

Email: Iotus1024@tom.com (8610)62283459

Wang Cong, a professor in CITSR in Beijing University of Posts and Telecommunications, engages in research on Cybernetics and Intelligence science for years.

Email: wangc@bt-t.com (8610) 62283459

Lin Jie, a postgraduate in CITSR (Center for Intelligence Technology and Science Research) in Bejing University of Posts and Telecommunications, major in Cybernetics and Intelligence science, and at present, focus on Network techrology and Information Systems.

Email: lotus1024@tom.com (8610)62286100 